\title{
Measuring hemodynamics in the developing heart tube with four-dimensional gated Doppler optical coherence tomography
}

\author{
Michael W. Jenkins \\ Case Western Reserve University \\ Department of Pediatrics \\ Cleveland, Ohio 44106
}

\author{
Lindsy Peterson \\ Shi Gu \\ Madhusudhana Gargesha \\ David L. Wilson \\ Case Western Reserve University \\ Department of Biomedical Engineering \\ Cleveland, Ohio 44106
}

\section{Michiko Watanabe}

Case Western Reserve University

Department of Pediatrics

Cleveland, Ohio 44106

\section{Andrew M. Rollins}

Case Western Reserve University

Department of Biomedical Engineering

Cleveland, Ohio 44106

\begin{abstract}
Hemodynamics is thought to play a major role in heart development, yet tools to quantitatively assess hemodynamics in the embryo are sorely lacking. The especially challenging analysis of hemodynamics in the early embryo requires new technology. Small changes in blood flow could indicate when anomalies are initiated even before structural changes can be detected. Furthermore, small changes in the early embryo that affect blood flow could lead to profound abnormalities at later stages. We present a demonstration of 4-D Doppler optical coherence tomography (OCT) imaging of structure and flow, and present several new hemodynamic measurements on embryonic avian hearts at early stages prior to the formation of the four chambers. Using 4-D data, pulsed Doppler measurements could accurately be attained in the inflow and outflow of the heart tube. Also, by employing an enface slice from the 4-D Doppler image set, measurements of stroke volume and cardiac output are obtained without the need to determine absolute velocity. Finally, an image plane orthogonal to the blood flow is used to determine shear stress by calculating the velocity gradient normal to the endocardium. Hemodynamic measurements will be crucial to identifying genetic and environmental factors that lead to congenital heart defects. () 2010 Society of Photo-Optical Instrumentation Engineers. [DOI: 10.1117/1.3509382]
\end{abstract}

Keywords: hemodynamics; embryo; optical coherence tomography 4-D Doppler imaging; congenital heart defect.

Paper 10455LR received Aug. 13, 2010; revised manuscript received Oct. 5, 2010; accepted for publication Oct. 7, 2010; published online Dec. 13, 2010.

\section{Introduction}

Blood flow is vital for nutrient and $\mathrm{O}_{2}$ delivery to tissue, but also influences gene expression through mechanical stimulation, which is crucial in the developmental process in the early embryonic heart. ${ }^{1,2}$ Identifying the genesis and following steps leading to congenital heart defects has been difficult. Closely monitoring blood flow in early development may provide clues to identifying the etiology of congenital heart defects. For example, certain septal defects in the four-chambered heart may originate from improper gene expression caused by altered blood flow-induced shear stresses on the endocardium in the tubular heart. ${ }^{1}$ Because of the importance of blood flow in regulating heart development, it is essential to develop tools to monitor and analyze hemodynamics.

Optical coherence tomography (OCT) has shown great potential in imaging the developing heart. ${ }^{3-9}$ Many cardiac OCT measurements can now be used to assess embryonic heart function. In particular, hemodynamic measurements possess the potential to accurately measure blood velocity, blood flow, and shear stress on the endocardium of the developing heart tube. Davis et al. made pulsed Doppler measurements in the outflow tract of early-stage chicken embryos by taking M-scans. ${ }^{4}$ The

Address all correspondence to: Michael W. Jenkins, Department of Pediatrics, Case Western Reserve University, 10900 Euclid Ave., Cleveland, OH 44106 Tel: 1216 844-3298; E-mail: mwj5@case.edu. shape of pulsed Doppler OCT waveforms are independent of the absolute velocity of blood flow, and therefore do not depend on the incident angle of OCT illumination. This simplifies their use for analyzing cardiac function. Measuring pulsed Doppler in the heart using M-scans can be difficult to align due to the constant heart movement and the inability to visualize the heart in three dimensions. By taking a 4-D dataset, not only can one assure pulsed Doppler measurements from the middle of the heart tube, but multiple measurements can be made along the tube. The pulsed Doppler waveform at the inflow of the heart tube differs greatly from the outflow waveform. Davis et al. also indirectly measured flow and shear rate in the vitelline vessels of a chick embryo. ${ }^{6}$ However, 4-D imaging is needed to make direct measurements of flow and shear rate in the 3-D complex heart tube. Venneman et al. measured shear stress in the embryonic avian heart using microparticle image velocimetry ( $\mu$ PIV) on a series of 2-D images. ${ }^{10}$ To analyze the effect of shear stress on the entire endocardium, a volumetric dataset is needed to accurately assess the velocity gradient normal to the heart wall. Recently, we developed gated OCT imaging to image the embryonic heart in 4-D. ${ }^{7}$ Here, we present 4-D images of blood flow that enable hemodynamic measurements within the heart.

We demonstrate hemodynamic measurements made on an embryonic avian heart using 4-D Doppler OCT data, in which

$1083-3668 / 2010 / 15(6) / 066022 / 4 / \$ 25.00$ (C) 2010 SPIE 
the velocity data have been unwrapped using a Goldstein algorithm. ${ }^{6,11}$ Using 4-D data, pulsed Doppler measurements were obtained at several locations within the heart tube simultaneously to assess regions of interest. Also, by enabling measurement of blood flow through an en-face slice, 4-D Doppler OCT allows the measurement of stroke volume and cardiac output without the need to determine absolute velocity. Finally, 4-D Doppler OCT makes possible the selection of image planes orthogonal to the blood flow to determine shear stress by calculating the velocity gradient normal to the endocardium.

\section{Methods}

To acquire 4-D Doppler OCT images, fertilized quail eggs were incubated in a humidified, forced draft incubator at $38^{\circ} \mathrm{C}$. At $48 \mathrm{~h}$ of development, eggs were taken out of the incubator, the eggshells were removed, and the contents were cultured in sterilized 3.5-cm-diam Petri dishes inside an environmentally controlled OCT imaging chamber. The imaging chamber controlled temperature $\left(38^{\circ} \mathrm{C}\right)$ and humidity are essential to ensure imaging under near-physiological conditions. The OCT system employed a buffered Fourier domain mode locked (FDML)-laser ${ }^{3}$ operating at a $117-\mathrm{kHz}$ line rate. The resolution (axial and lateral) of the system was $\sim 10 \mu \mathrm{m}$ in air. 4-D gated datasets were collected by imaging over multiple heartbeats at each slice location, and assembling the data in postprocessing, as described previously. ${ }^{7}$ All final datasets consisted of 60 volumes per heartbeat. To accommodate Doppler imaging, OCT A-scans were recorded at $1.3-\mu \mathrm{m}$ steps in the B-scan direction. A fiveline rolling average was applied to the Doppler data to reduce noise. A calibration interferometer was used both to resample the data evenly in wave number and to improve the Doppler signal by correcting for small fluctuations in sweep progression. A Goldstein algorithm was utilized to unwrap the Doppler data. ${ }^{6,11}$ Signal processing and image visualization was carried out using Matlab (The MathWorks, Natick, Massachusetts) and Amira (Visage Imaging GmbH, Berlin, Germany). We obtained pulsed Doppler waveforms from arbitrary locations in the heart tube by selecting fixed small windows $(5 \times 5$ pixels $)$ at approximately the center of the tube at locations of interest along the tube. The pixels in the window were utilized to compute the pulsed Doppler waveform in the same way as using a line segment in pulsed Doppler ultrasound.

We measured stroke volume as the volume of blood pumped by one heart beat, and cardiac output as blood volume pumped per unit time. Stroke volume and cardiac output can be obtained by computing the flow past a defined plane over a heart cycle, which can be achieved using a 4-D Doppler dataset. The velocity component perpendicular to the slice is needed to compute flow. By choosing an en-face slice, the perpendicular velocity component is directly measured by the OCT system and there is no need to find the absolute velocity. ${ }^{12}$ Unlike measurements of shear stress in the heart, stroke volume and cardiac output can be easily obtained from a group of en-face slices without the laborious task of correcting the velocity for angle of incidence. The velocity profile was integrated over the area of the lumen to yield flow, and flow was integrated over time to yield flow per heart beat or stroke volume. Cardiac output was calculated by multiplying stroke volume and heart rate.
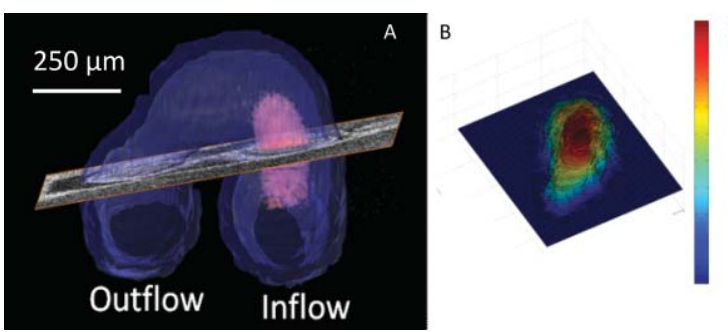

$20 \mathrm{~mm} / \mathrm{s}$

Fig. 1 Calculating shear stress. (a) The segmented heart is indicated in blue and the blood flow in the inflow in pink. An oblique slice is positioned in an orthogonal orientation to the blood flow to calculate shear stress. (b) Velocity profile was obtained from the Doppler OCT data at the oblique cross section selected in (a) and is displayed as a surface plot.

We calculated shear stress as $\tau=\eta d u / d n$, where $\eta$ is the dynamic viscosity of the blood and $d u / d n$ is the wall-normal velocity gradient or the shear rate. Here, the blood is assumed to be Newtonian (no-slip condition at the endocardium). ${ }^{10}$ Blood flow in the looping avian heart is dominated by viscous forces and is laminar, not turbulent, due to a low Reynolds and Womersley number. ${ }^{10}$ Like previous $\mu$ PIV measurements of shear stress, the dynamic viscosity was approximated at $5 \mathrm{mPa} \mathrm{s} .{ }^{10}$ To calculate the velocity normal to the endocardium, oblique slices normal to the direction of flow were manually selected [see Fig. 1(a)] at specific time points by observing both the structural and Doppler data. The velocity was corrected for angle of incidence by calculating the angle between the perpendicular slice normal and the incident light vector. For each observed time point, the oblique slices normal to the blood flow and angle of incidence were recalculated. Next, the blood endocardium interface was identified using the structural images. The Doppler image was smoothed using a median filter, and the velocity profile normal to the endocardium [see Fig. 1(b)] was employed to calculate $d u / d n$. The velocity gradient was computed utilizing the linear portion (approximately $25 \mu \mathrm{m}$ ) of the velocity profile adjacent to the wall. Finally, shear stress was computed by multiplying $d u / d n$ and dynamic viscosity.

\section{Results and Discussion}

For this demonstration, two stage-14 quail embryos were imaged as described before. At this stage, the heart is a looped tube where the caudal portion of the tube is beginning to move cranially. ${ }^{13}$ Figure 2 shows pulsed waveforms collected from the inflow and outflow of one of the stage-14 quail embryos. The 4-D dataset allows one to choose multiple points along the heart tube to examine cardiac function. The shape of the waveform changes significantly from the inflow to the outflow of the developing tube. The inflow pulse has the two peaks characteristic of venous flow, while the outflow has a single peak characteristic of arterial flow. ${ }^{14}$ When observing cardiac anomalies, pulsed waveforms from both the inflow and outflow may allow investigators a more complete picture of the mechanism underlying the anomaly. For example, large retrograde flow in the outflow of the tube may be caused by poor filling in the inflow. Although the same data can be collected by performing two separate M-scans, with the heart in constant motion it is difficult to select the appropriate region to obtain the pulsed Doppler measurement. A 4-D dataset 


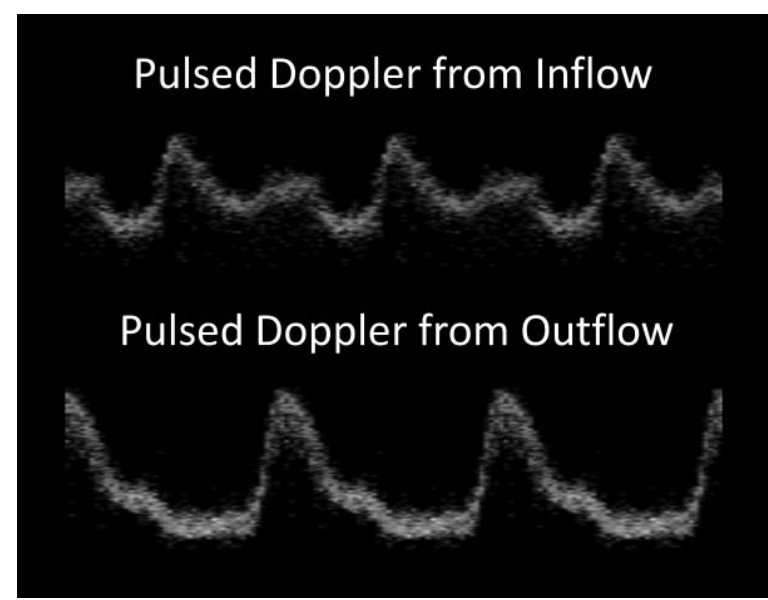

Fig. 2 Pulsed Doppler. The pulsed Doppler waveform at the inflow of the heart tube is distinctly different from the outflow waveform. The 4-D Doppler dataset enabled us to obtain pulsed Doppler traces at any location along the heart tube, which allowed easy access to multiple areas of interest. 4-D Doppler also overcomes the difficulty in selecting appropriate regions to perform $\mathrm{M}$-scans in the beating heart. The top panel represents a waveform taken from the inflow of the heart tube, while the bottom panel is from the outflow of the heart tube. The inflow pulse has the two peaks characteristic of venous flow, while the outflow has a single peak characteristic of arterial flow.

allows one to select a pulsed Doppler signal from a location that maintains a position near the center of the lumen throughout the heartbeat, with minimal distortion of the waveform.

Video 1 shows a volume rendering of blood flowing through the heart tube over a heart cycle. Color Doppler is overlaid on the

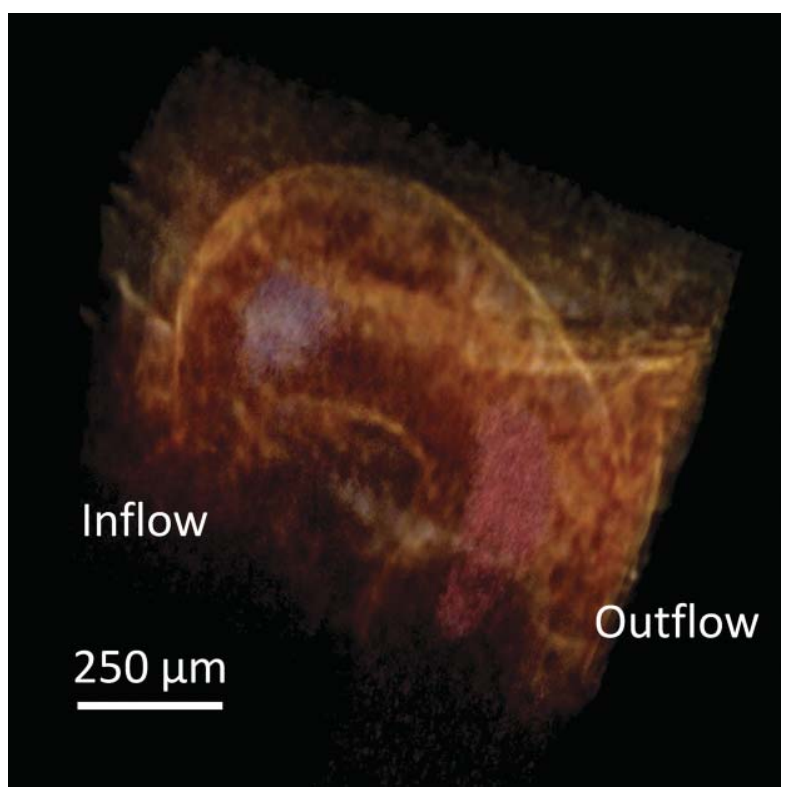

Video 1 4-D Doppler OCT image of blood flow. Doppler data are superimposed on a 3-D structure of the heart. Blue indicates blood flow toward the illumination beam, while red indicates flow away from the beam. The video also includes the pulsed Doppler traces from Fig. 2 with a scrolling line matching the phase of the pulsed Doppler waveform with the corresponding cardiac volume. The video allows one to visualize the interplay between movement of the heart walls and blood flow.

[URL: http://dx.doi.org/10.1117/1.3509382.1]
Table 1 Hemodynamic measurements from two stage-14 quail embryos.

\begin{tabular}{lcc}
\hline & Embryo 1 & Embryo 2 \\
\hline Stage & 14 & 14 \\
Heart rate (BPM) & 182 & 195 \\
Max velocity inflow $(\mathrm{mm} / \mathrm{s})$ & 26.2 & 18.1 \\
Max velocity oufflow $(\mathrm{mm} / \mathrm{s})$ & 51 & 58.5 \\
Stroke volume $(\mu \mathrm{L})$ & 0.04 & 0.038 \\
Cardiac output $(\mu \mathrm{L} / \mathrm{s})$ & 0.123 & 0.124 \\
Max shear rate inflow $(1 / \mathrm{s})$ & 693.3 & 417.4 \\
Max shear Stress inflow $(\mathrm{Pa})$ & 3.5 & 2.1 \\
Max shear rate oufflow $(1 / \mathrm{s})$ & 1521 & 1834 \\
Max shear stress oufflow $(\mathrm{Pa})$ & 7.6 & 9.2 \\
\hline
\end{tabular}

structural image, where blue represents blood moving toward the incident beam of light and red represents blood moving away. The movie also includes the pulsed Doppler traces from the inflow and outflow with a scrolling line matching the phase of the pulsed Doppler waveform with the corresponding cardiac volume. The movie allows one to visualize the interplay between movement of the heart walls and blood flow.

In Table 1, the hemodynamics of the two stage-14 quail embryo hearts are summarized quantitatively. Heart rate, stage, and maximum velocity are presented for each heart. The maximum velocity measurement is consistent with echocardiography measurements of maximum velocity on similar stage embryos (data from collaborator Linask not shown). ${ }^{15}$ 4-D image sets allowed us to measure velocity along the heart tube. The average cardiac output and stroke volume of the two stage-14 hearts was $0.123 \mu \mathrm{L} / \mathrm{s}$ and $0.039 \mu \mathrm{L}$. The average maximum shear rates and stresses were higher in the outflow (1678 1/s and $8.4 \mathrm{~Pa})$ than the inflow $(5551 / \mathrm{s}$ and $2.8 \mathrm{~Pa}$ ) of the heart tube. The maximum shear stress we report is slightly higher than previous measurements using $\mu \mathrm{PIV}(5 \mathrm{~Pa})$ on stage- 15 chick embryos, ${ }^{10}$ which may be due to the fact that OCT could measure shear stress on volumetric data.

\section{Conclusions}

We present 4-D OCT Doppler imaging of an embryonic heart. The Doppler data are unwrapped using a Goldstein algorithm. ${ }^{6,11}$ The 4-D data allow us to examine hemodynamic differences between the inflow and outflow of the heart tube (maximum velocity, shear rate, etc.). Pulsed Doppler waveforms show significantly different hemodynamic measurements at different locations along the heart tube. 4-D Doppler also overcomes the difficulty in selecting appropriate regions to perform M-scans in the beating heart. Also, we use a simple method to measure cardiac output and stroke volume that is enabled by a 4-D image set. Finally, the 4-D Doppler data allow us to select orthogonal slices to the heart tube and compute shear rate and 
shear stress. With this method we will be able to collect typical hemodynamic levels in embryos over several key stages of development to establish what constitutes normal versus abnormal heart development.

\section{Acknowledgments}

This research is supported in part by the NIH (RO1-HL083048, RO1 HL095717, and T32HD007104). This investigation was conducted in a facility constructed with support from the Research Facilities Improvement Program grant number C06 RR12463-01 from the National Center of Research Resources, NIH. We would like to thank Kersti Linask for sharing echocardiography data for comparing the OCT data.

\section{References}

1. J. R. Hove, R. W. Koster, A. S. Forouhar, G. Acevedo-Bolton, S. E. Fraser, and M. Gharib, "Intracardiac fluid forces are an essential epigenetic factor for embryonic cardiogenesis," Nature 421 (6919), 172177 (2003).

2. B. C. W. Groenendijk, B. P. Hierck, J. Vrolijk, M. Baiker, M. J. Pourquie, A. C. Gittenberger-de Groot, and R. E. Poelmann, "Changes in shear stress-related gene expression after experimentally altered venous return in the chicken embryo," Circ. Res. 96(12), 12911298 (2005).

3. M. W. Jenkins, D. C. Adler, M. Gargesha, R. Huber, F. Rothenberg, J. Belding, M. Watanabe, D. L. Wilson, J. G. Fujimoto, and A. M. Rollins, "Ultrahigh-speed optical coherence tomography imaging and visualization of the embryonic avian heart using a buffered Fourier domain mode Locked laser," Opt. Express 15(10), 6251-6267 (2007).

4. A. Davis, F. G. Rothenberg, N. Shepherd, and J. A. Izatt, "In vivo spectral domain optical coherence tomography volumetric imaging and spectral Doppler velocimetry of early stage embryonic chicken heart development," J. Opt. Soc. Am. A 25, 3134-3143 (2008).
5. S. Rugonyi, C. Shaut, A. Liu, K. Thornburg, and R. K. Wang, "Changes in wall motion and blood flow in the outflow tract of chick embryonic hearts observed with optical coherence tomography after outflow tract banding and vitelline-vein ligation," Phys. Med. Biol. 53, 5077-5091 (2008).

6. A. Davis, J. Izatt, and F. Rothenberg, "Quantitative measurement of blood flow dynamics in embryonic vasculature using spectral Doppler velocimetry," Anat. Rec. 229, 311-319 (2009).

7. M. Gargesha, M. W. Jenkins, D. L. Wilson, and A. M. Rollins, "High temporal resolution OCT using image-based retrospective gating," Opt. Express 17(13), 10786-10799 (2009).

8. I. V. Larina, S. Ivers, S. Syed, M. E. Dickinson, and K. V. Larin, "Hemodynamic measurements from individual blood cells in early mammalian embryos with Doppler swept source OCT," Opt. Lett. 34(7), 986-988 (2009)

9. J. Manner, L. Thrane, K. Norozi, and T. M. Yelbuz, "In vivo imaging of the cyclic changes in cross-sectional shape of the ventricular segment of pulsating embryonic chick hearts at stages 14 to 17: a contribution to the understanding of the ontogenesis of cardiac pumping function," Dev. Dyn. 238(12), 3273-3284 (2009).

10. P. Vennemann, K. T. Kiger, R. Lindken, B. C. Groenendijk, S. Stekelenburg-de Vos, T. L. ten Hagen, N. T. Ursem, R. E. Poelmann, J. Westerweel, and B. P. Hierck, "In vivo micro particle image velocimetry measurements of blood-plasma in the embryonic avian heart," J. Biomech. 39, 1191-1200 (2006).

11. R. M. Goldstein, H. A. Zebker, and C. L. Werner, "Satellite radar interferometry-two-dimensional phase unwrapping," Radio Sci. 23(4), 713-720 (1988).

12. V. J. Srinivasan, S. Sakadzic, I. Gorczynska, S. Ruvinskaya, W. Wu, J. G. Fujimoto, and D. A. Boas, "Quantitative cerebral blood flow with optical coherence tomograph," Opt. Express 18(3), 2477-2494 (2010).

13. A. L. Romanoff, "The heart," in The Avian Embryo, pp. 683-692, The Macmillan Co., New York (1960).

14. G. B. Chavhan, D. A. Parra, A. Mann, and O. M. Navarro, "Normal Doppler spectral waveforms of major pediatric vessels: specific patterns," Radiograph. 28(3), 691-706 (2008).

15. K. K. Linask, "Echocardiography (echo) of a HH stage 13 avian heart," Univ. South Florida, St. Petersburg (2009). 